PROCEEDINGS OF THE

AMERICAN MATHEMATICAL SOCIETY

Volume 129, Number 3, Pages 719-724

S 0002-9939(00)05944-X

Article electronically published on November 8, 2000

\title{
A TWO-DIMENSIONAL HAHN-BANACH THEOREM
}

\author{
B. L. CHALMERS AND B. SHEKHTMAN \\ (Communicated by Dale Alspach)
}

\begin{abstract}
Let $\tilde{T}=\sum_{i=1}^{n} \tilde{u}_{i} \otimes v_{i}: V \rightarrow V=\left[v_{1}, \ldots, v_{n}\right] \subset X$, where $\tilde{u}_{i} \in V^{*}$ and $X$ is a Banach space. Let $T=\sum_{i=1}^{n} u_{i} \otimes v_{i}: X \rightarrow V$ be an extension of $\tilde{T}$ to all of $X$ (i.e., $u_{i} \in X^{*}$ ) such that $T$ has minimal (operator) norm. In this paper we show in particular that, in the case $n=2$ and the field is $\mathbf{R}$, there exists a rank- $n \tilde{T}$ such that $\|T\|=\|\tilde{T}\|$ for all $X$ if and only if the unit ball of $V$ is either not smooth or not strictly convex. In this case we show, furthermore, that, for some $\|T\|=\|\tilde{T}\|$, there exists a choice of basis $v=v_{1}, v_{2}$ such that $\left\|u_{i}\right\|=\left\|\tilde{u}_{i}\right\|, i=1,2$; i.e., each $u_{i}$ is a Hahn-Banach extension of $\tilde{u}_{i}$.
\end{abstract}

\section{INTRODUCTION}

Let $X$ be a Banach space and $V$ be a subspace of $X$. If $T$ is an operator from $X$ into $V$, then $T_{\mid}$is the restriction of $T$ onto $V$. Let $\tilde{T}: V \rightarrow V$.

Define

$$
e(\tilde{T} ; X)=\inf _{T}\left\{\|T\|: T_{\mid V}=\tilde{T}\right\}
$$

and

$$
e(\tilde{T})=\sup _{X}\{e(\tilde{T}: X): X \supset V\} .
$$

The classical Hahn-Banach Theorem states that, for every rank-one operator $\tilde{T}$,

$$
e(\tilde{T})=\|\tilde{T}\| .
$$

The Nachbin Theorem shows that, if $V$ is $n$-dimensional and, for every $\tilde{T}: V \rightarrow V$,

$$
e(\tilde{T})=\|\tilde{T}\|,
$$

then $V \cong \ell_{\infty}^{(n)}$.

Definition 1.1. An operator $\tilde{T}: V \rightarrow V$ is called a Hahn-Banach operator if $e(\tilde{T})=\|\tilde{T}\|$.

In this paper we are concerned with the existence of non-trivial Hahn-Banach operators on a Banach space $V$. In particular we show (Theorem 2.2) that the two-dimensional real Banach space $V$ possesses a rank-two Hahn-Banach operator if and only if $V$ is either non-smooth or not strictly convex.

For the proof of the theorem we will need a number of definitions.

Received by the editors January 5, 1999

2000 Mathematics Subject Classification. Primary 46B20; Secondary 47A20.

(C)2000 American Mathematical Society 
Let $X$ be a Banach space and $V$ be a finite-dimensional subspace. Let $T$ be a bounded linear operator from $X$ into $V$ and let $\tilde{T}$ be the restriction of $T$ onto $V$. $\left(\tilde{T}=T_{\left.\right|_{V}}\right)$

Definition 1.2. $T$ is a minimal norm extension of $\tilde{T}$ means

$$
\|T\|=\inf \left\{\|S\|: S: X \rightarrow V:\left.S\right|_{V}=\tilde{T}\right\} .
$$

Definition 1.3. The extremal set of $T: X \rightarrow V$ is

$$
\mathcal{E}(T)=\left\{\left(x^{*}, x^{* *}\right) \in B\left(X^{*}\right) \times B\left(X^{* *}\right): x^{*}\left(T^{* *} x^{* *}\right)=\|T\|\right\},
$$

where $B$ denotes the unit ball and $T^{* *}$ denotes the second adjoint extension of $T$ $\left(T^{* *} x^{* *}=\sum_{i=1}^{n}\left\langle u_{i}, x^{* *}\right\rangle v_{i}\right.$ and $\left.\left\|T^{* *}\right\|=\|T\|\right)$.

Note 1.1. In the following we will designate by $x^{*} \otimes x^{* *}: X \rightarrow X^{* *}$ the usual dyad operator given by $x^{*} \otimes x^{* *}(x)=\left\langle x, x^{*}\right\rangle x^{* *}$.

Theorem $1.1([2])$. $T$ is a minimal norm extension of $\tilde{T}$ if and only if there exists a probability measure $\mu$ on $\mathcal{E}(T)$ such that the operator

$$
E_{T}=\int x^{*} \otimes x^{* *} d \mu
$$

maps $V$ into $V$.

Definition 1.4. An $n$-dimensional subspace $V \subset L_{1}[\mathcal{T}, \nu]$ is said to be smooth if no non-zero element $v$ of $V$ vanishes on a set of positive $\nu$-measure.

Note 1.2. It is well known that the $n$-dimensional real space $V$ is smooth if and only if the unit sphere $S(V)$ is differentiable (has a unique $(n-1)$-dimensional tangent plane) at every point. This fact follows immediately from $\|\vec{a}\|:=\|\vec{a} \cdot \vec{v}\|$ and the formula (see e.g. [1])

$$
\frac{\partial}{\partial a_{i}}\|\vec{a}\|=\int_{T} v_{i}(s) \operatorname{sgn}[\vec{a} \cdot \vec{v}(s)] d \nu(s) \text { for } \vec{a} \neq 0
$$

and the Implicit Function Theorem. A point where $S(V)$ is non-differentiable is called a point of non-smoothness.

Theorem $1.2([\mathbb{1}])$. Let $V=[\vec{v}]$ be a smooth real subspace of $L_{1}[\mathcal{T}, \nu]$. Then $T=\sum_{i=1}^{n} u_{i} \otimes v_{i}$ is a minimal extension of $\tilde{T}\left(\right.$ to $\left.L_{1}[\mathcal{T}, \nu]\right)$ implies that, for almost all $t$,

$$
\vec{u}(t)=\|T\| \vec{z}(t)
$$

with $\vec{z}(t)$ being a point of intersection of $S(V)$ and its tangent plane perpendicular to $M \vec{v}(t)$, where $M$ is the $n \times n$ matrix $M$ of $\left(E_{T}\right)_{\left.\right|_{V}}$ with respect to the basis $\vec{v}$. (See Figure 1.)

Corollary 1.1. Let $V=[\vec{v}]$ be a smooth real subspace of $L_{1}[\mathcal{T}, \nu]$. Then without loss $\mathcal{T}$ can be enlarged so that there exists $T=\sum_{i=1}^{n} u_{i} \otimes v_{i}$ which is a minimal extension of $\tilde{T}$ and the operator $T^{*}$ is an extension of $\tilde{T}^{*}: V^{*} \rightarrow V^{*}$.

Proof. Let $T=\sum_{i=1}^{n} u_{i} \otimes v_{i}$ be a minimal extension of $\tilde{T}$. Theorem 1.2 provides that $\vec{u} /\|T\|$ lies on $S(V)$ a.e. $[\nu]$. Next replace $\mathcal{T}$ by $\mathcal{T} \cup \Delta \mathcal{T}$, where $\Delta \mathcal{T}=S(V)$-range $(\vec{u} /\|T\|)$, enlarge $\nu$ and $\vec{v}$ to be zero on $\Delta \mathcal{T}$, and enlarge $\vec{u} /\|T\|$ to be the coordinate functions on $\Delta \mathcal{T}$. Then let $T$ and $\tilde{T}$ be replaced by their corresponding enlargements, recall that $V^{*}$ is isometric to the space of coordinate functions of $S(V)$ regarded as a subspace of $L_{\infty}(S(V))$, and note that $T_{\left.\right|_{V^{*}} ^{*}}=\tilde{T}^{*}$. 


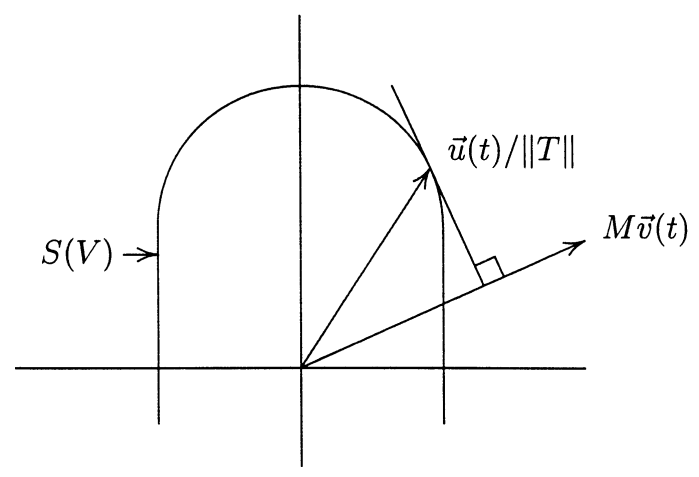

FIGURE 1.

\section{MAIN THEOREM}

Lemma 2.1. Let $T$ be an extension of $\tilde{T}$. Then $\|T\|=\|\tilde{T}\|$ if and only if there exists a pair

$$
\left(x^{*}, v\right) \in \mathcal{E}(T), v \in V .
$$

Hence the dyad operator $E_{T}=x^{*} \otimes v$ satisfies the conclusion of Theorem 1.1.

Proof. If $\left(x^{*}, v\right) \in \mathcal{E}(T)$, then

$$
\|T\|=x^{*}(T v)=\|T v\| .
$$

Thus $T$ attains its norm on $V$ and

$$
\|T\|=\|T v\|=\|\tilde{T} v\| \leq\|\tilde{T}\| .
$$

Conversely, if $\|T\|=\|\tilde{T}\|$, let $v \in V$ be such that $\|\tilde{T} v\|=\|T v\|=\|\tilde{T}\|=\|T\|$. Let $x^{*} \in X^{*}$ be such that $x^{*}(T v)=\|T v\|$. Then $\left(x^{*}, v\right) \in \mathcal{E}(T)$.

The following theorem shows that, if $n>1$ and the field is $\mathbf{R}$, a smooth, strictly convex, $n$-dimensional $L_{1}$-subspace $V$ possesses no rank- $n$ Hahn-Banach operator. In fact it shows more, namely, that $V$ possesses no rank- $k$ operator which extends to $L_{1}$ of the same norm, $k>1$.

Theorem 2.1. Let $V$ be a smooth, strictly convex, $n$-dimensional real subspace of $L^{1}[-1,1]$. Let $\tilde{T}: V \rightarrow V$ be such that there exists an extension $T: L^{1}[-1,1] \rightarrow V$ with $\|T\|=\|\tilde{T}\|$. Then $\tilde{T}$ is a rank-one operator.

Proof. By Lemma 2.1, corresponding to $T=\sum_{i=1}^{n} u_{i} \otimes v_{i}$, there exists a rank-one operator $E_{T}$ satisfying the conclusion of the lemma. Hence the matrix $M$ defined as the matrix of $\left(E_{T}\right)_{\left.\right|_{V}}$, with respect to the basis $\vec{v}$, has rank one.

Since $V$ is smooth there exists a minimal extension $T$ of $\tilde{T}$ satisfying the conditions of Theorem 1.2 But $M$ has rank one, whence the vectors $M \vec{v}(t)$ lie on the same one-dimensional subspace of $\mathbf{R}^{n}$ for almost all $t \in[-1,1]$.

Since $V$ is strictly convex, the vectors $\vec{u}(t)$ also lie in the same one-dimensional subspace of $\mathbf{R}^{n}$ for almost all $t \in[-1,1]$. Hence the operator $T$ given by Theorem 1.2 has rank one and thus so does $\tilde{T}$.

Corollary 2.1. $n$-dimensional Hilbert space $\left(\ell_{2}^{(n)}\right)$ possesses no Hahn-Banach operator with rank $>1$. 
Proof. It is well known (see [5]) that $\ell_{2}^{(n)}$ can be realized as a subspace of $L_{1}$.

Theorem 2.2. Let $V$ be a two-dimensional real Banach space. Then the following are equivalent:

(1) $V$ is smooth and strictly convex.

(2) For every rank-two operator

$$
\tilde{T}: V \rightarrow V, \quad e(\tilde{T})>\|\tilde{T}\| .
$$

Proof. (1) implies (2) by Theorem 2.1 since every two-dimensional space $V$ can be isometrically imbedded into $L_{1}[-1,1]$ (see e.g. 6]).

We now show (2) implies (1).

Suppose that $V$ with unit sphere $S(V) \subset \mathbf{R}^{2}$ is not strictly convex. Then (via a linear transformation) we can assume that $S(V)$ lies inside the unit square $C$, touches $C$ on all four sides, and has a flat spot running from $(1, d)$ to $(1,-d)$ (and of course a corresponding flat spot running from $(-1,-d)$ to $(-1, d)$ ), where "flat spot" is defined in the usual obvious way. Let $P$ be the rectangle with corners $( \pm 1, \pm d)$, whence $P$ lies inside $S(V)$. (See Figure A.)

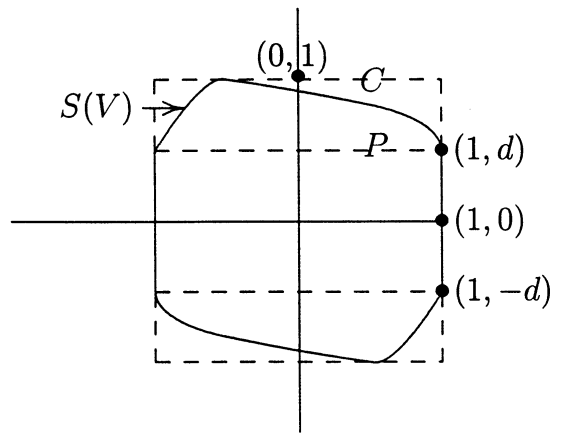

Figure A.

Now consider the linear transformation $\tilde{T}\left(a_{1}, a_{2}\right):=\left(a_{1}, d a_{2}\right)$ taking $C$ into $P$. Thus $\tilde{T}(S(V))$ lies inside $P$, which lies inside $S(V)$, and, since $\tilde{T}(1,0)=(1,0) \in$ $S(V) \cap \tilde{T}(S(V))$, we see that $\tilde{T}: V \rightarrow V$ has norm one. Note that we can also write $\tilde{T}$ in the form

$$
\tilde{T}=\tilde{\phi}_{1} \otimes(1,0)+d \tilde{\phi}_{2} \otimes(0,1),
$$

where $\tilde{\phi}_{i}$ are the norm- 1 coordinate functionals on $V$ defined by

$$
\tilde{\phi}_{i}\left(a_{1}, a_{2}\right)=a_{i}, i=1,2 .
$$

Next let $\phi_{i}$ be a Hahn-Banach extension to $X$ of the functional $\tilde{\phi}_{i}$ on $V, i=1,2$. Then the extension operator $T: X \rightarrow V$ has the form

$$
T=\phi_{1} \otimes(1,0)+d \phi_{2} \otimes(0,1) .
$$

Finally, since $T(S(X)) \subset \tilde{T}(\overline{c o} C)=\overline{c o} P \subset \overline{c o} S(V)=B(V)$, we see that $\|T\| \leq 1$ and hence, since $\|T\| \geq\|\tilde{T}\|=1$, it follows that $\|T\|=\|\tilde{T}\|$.

Furthermore, suppose that $V$ is strictly convex but not smooth. Then, as is well known, $V^{*}$ is smooth but not strictly convex, and the above argument shows that $V^{*}$ possesses a rank-two Hahn-Banach operator. But then, by Corollary 1.1 
$\left(1=\left\|T^{*}\right\|=\left\|\tilde{T}^{*}\right\|=\|\tilde{T}\|=\|T\|\right.$ and $\left.e(\tilde{T})=e\left(\tilde{T} ; L_{\infty}\right)\right), V$ also possesses a rank-two Hahn-Banach operator.

Corollary 2.2. If the unit ball of the 2-dimensional real space $V$ is either not smooth or not strictly convex, then $V$ possesses a basis $v_{1}, v_{2}$ and a rank-2 HahnBanach operator $\tilde{T}=\sum_{i=1}^{2} \tilde{u}_{i} \otimes v_{i}$ such that, for the extension $T=\sum_{i=1}^{2} u_{i} \otimes v_{i}$, each $\left\|u_{i}\right\|=\left\|\tilde{u}_{i}\right\|, i=1,2$, i.e., each $u_{i}$ is a Hahn-Banach extension of $\tilde{u}_{i} . A$ reasonable conjecture is that this phenomenon holds for every rank-2 Hahn-Banach operator on $V$.

Note 2.1. In [3] it is shown in particular that, for each real non-singular $2 \times 2$ real matrix $A$ not equal to a scalar multiple of the identity $I$, there exists a real space $V=[\vec{v}] \not \ell_{\infty}^{(2)}$ which possesses a Hahn-Banach operator $\tilde{T}$ such that $\tilde{T} \vec{v}=A \vec{v}$. Theorem 2.2 explains why any such space must be either non-smooth or non-strictly convex. Indeed all the spaces provided in 3 . are hexagonal spaces.

Note, furthermore, that the rectangle $P$ in the proof of Theorem 2.2 can be replaced by any parallelogram (symmetric with respect to the origin) contained inside $S(V)$ and intersecting the flat sides of $S(V)$. In this way we see that $V$ possesses an entire family of two-dimensional Hahn-Banach operators of norm-1 $(\tilde{T} p=p$ for some $p \in S(V) \cap \tilde{T}(S(V))$ of the form

$$
\tilde{T}=\left(\alpha \tilde{\phi}_{1}+\beta \tilde{\phi}_{2}\right) \otimes(1,0)+\left(\gamma \tilde{\phi}_{1}+\delta \tilde{\phi}_{2}\right) \otimes(0,1),
$$

all of which have two real eigenvalues (1 and $e$, where $|e| \leq 1)$. In 4 we show that, if the spectrum of $\tilde{T} \neq I$ lies on the unit circle, then the Hahn-Banach operator $\tilde{T}$ has norm $>1$.

Corollary 2.3. If the two-dimensional real Banach space $V$ possesses a rank-two Hahn-Banach operator $\tilde{T} \neq I$ whose spectrum lies on the unit circle, then $V$ also possesses a rank-two Hahn-Banach operator with eigenvalues $1, d,|d|<1$.

Corollary 2.4. The two-dimensional real Banach space $V$ possesses a rank-two Hahn-Banach operator if and only if its dual space $V^{*}$ possesses a rank-two HahnBanach operator.

Corollary 2.5. For every two-dimensional subspace $V$ of $\ell_{1}$ or $\ell_{\infty}$ there exists a rank-two operator $\tilde{T}: V \rightarrow V$ such that $e(\tilde{T})=\|\tilde{T}\|$.

For every two-dimensional subspace $V$ of $\ell_{p}(p \in(1, \infty))$ and for every rank-two operator $\tilde{T}: V \rightarrow V$ we have $e(\tilde{T})>\|\tilde{T}\|$.

Example 2.1. Let $V=[\vec{v}] \subset L^{1}[-1,1]$, where $\left(v_{1}, v_{2}\right)=\left(\frac{1}{2}, t\right)$ and let $\tilde{T} v_{1}=v_{1}$ and $\tilde{T} v_{2}=a v_{2}, a \geq \frac{1}{2}$. Then Theorem 1.2 yields that the minimal extension operator $T=\sum_{i=1}^{2} u_{i} \otimes v_{i}: L^{1}[-1,1] \rightarrow V$ is given by

$$
\vec{u}=\kappa\left[\frac{(1,2 m t)}{\sqrt{1+4 m^{2} t^{2}}}+\left(0, \frac{\operatorname{sgn} t}{2}\right)\right],
$$

where $\kappa=\|T\|, m$ is a non-negative constant, and $M=\left(\begin{array}{ll}1 & 0 \\ 0 & m\end{array}\right)$. (In fact $a=$ $\left(\frac{1}{2}+\int_{0}^{1} \frac{4 m t^{2} d t}{\sqrt{1+4 m^{2} t^{2}}}\right) / \int_{0}^{1} \frac{d t}{\sqrt{1+4 m^{2} t^{2}}}$. $)$ Since $V$ is smooth, Theorem 2.2 provides that $\|T\|=\|\tilde{T}\|=1$ precisely when $m=0$. Note that then $u_{1}=1$ and $u_{2}=\frac{1}{2} \operatorname{sgn} t$ shows that $u_{i} \in L_{\infty}[-1,1]$ is the Hahn-Banach extension of $\tilde{u}_{i}, i=1,2$. (See Figure 2.) 


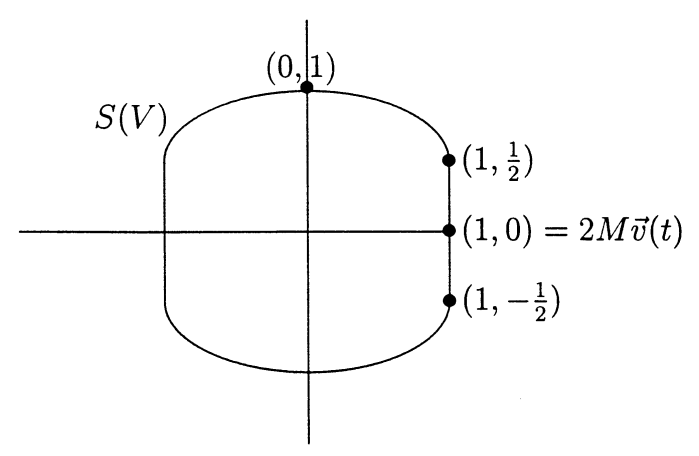

FIGURE 2.

As a further example, following Corollary 1.1, note that the space $V$ is also realized as $V=\left[\frac{1}{2}, t\right] \subset L^{1}[\overline{\mathbf{R}}, \nu]$, where $\nu$ is Lebesgue measure on $[-1,1]$ and zero off $[-1,1]$. Thus replacing $[-1,1]$ by $\overline{\mathbf{R}}$ in the above, we see that, for $m>0$, $\vec{u} /\|T\|$ now covers all of $S(V)$ and, for $m=0$, let $\vec{u}(t) /\|T\|=\vec{u}(t),|t|>1$, be any convenient parameterization of the curved portion of $S(V)$. Thus for $m=0$ the adjoint operator $\tilde{T}^{*}$ (with same-norm extension $T^{*}$ ) provides a Hahn-Banach operator for the non-smooth, strictly convex space $V^{*}=\left[t, 1-t^{2}\right] \subset L_{\infty}[-1,1]$ with a "corner" at $(1,0)$. $\left(V^{*} \cong[\vec{u} /\|T\|] \subset L_{\infty}[\overline{\mathbf{R}}].\right)$

\section{ACKNOWLEDGMENT}

The authors would like to thank Professor Mikhail Ostrovskii for his very valuable help with respect to this paper.

\section{REFERENCES}

[1] B. L. Chalmers and F. T. Metcalf, The determination of minimal projections and extensions in $L^{1}$, Trans. Amer. Math. Soc., 329(1992), 289-305. MR 92e:41017

[2] B. L. Chalmers and F. T. Metcalf, A characterization and equations for minimal projections and extensions, J. Operator Theory, 32(1994), 31-46. MR 96c:46014

[3] B. L. Chalmers and B. Shekhtman, Actions that characterize $\ell_{\infty}^{(n)}$, Lin. Alg. and Appl., 270(1998), 155-169. MR 98h:46008

[4] B. L. Chalmers and B. Shekhtman, Spectral properties of operators that characterize $\ell_{\infty}^{(n)}$, Abst. Appl. Anal. 3 (1998), 237-246. CMP 2000:11

[5] L. E. Dor, Potentials and isometric embeddings in $L_{1}$, Israel J. Math. 24(1976), 260-268. MR 54:5806

[6] D. Yost, $L_{1}$ contains every two-dimensional normed space, Ann. Polonici Math. 49(1988), 17-19. MR 90b:46047

Department of Mathematics, University of California, Riverside, California 92507

E-mail address: blc@math.ucr.edu

Department of Mathematics, University of South Florida, Tampa, Florida 33620

E-mail address: boris@math.usf.edu 